\title{
Clinical Outcome and Influencing Factor for Repeat Lumbar Discectomy for Ipsilateral Recurrent Lumbar Disc Herniation
}

\author{
Yeon Sung Jung, Hyu Jin Choi, Young-Min Kwon \\ Department of Neurosurgery, Dong-A University College of Medicine, Busan, Korea
}

Objective: Recurrent herniation following disc excision has been reported in $5-15 \%$ of patients. There have been numerous studies of recurrent disc herniation, but these have analyzed mixed patient populations. We designed this study to analyze the factors that influencing the clinical results, and efficiency of repeat discectomy for recurrent lumbar disc herniation occurring at the same level and on the same side after primary discectomy.

Methods: Between 1990 and 2011, a total of 52 patients who underwent repeat discectomy for recurrent lumbar disc herniation were retrospectively analyzed. Clinical outcomes were measured with Macnab criteria and visual analog scale (VAS score). Clinical parameters were also analyzed for influencing factors for outcome.

Results: Based on Macnab criteria, an excellent surgical outcome was achieved in 28 cases (54\%), a good outcome in 22 cases $(42 \%)$, a fair outcome in 1 case $(2 \%)$, and a poor outcome in 1 case $(2 \%)$. Based on VAS score, 47 of 52 patients $(90 \%)$ showed more than 4 score improvement. Age, sex, diabetes mellitus, smoking, time interval between repeat and primary discectomy, duration of recurrent symptoms, and extent of disc herniation did not significantly affect Macnab criteria and VAS score. However, a traumatic event showed less VAS score improvement whereas not affecting on Macnab criteria. Conclusions: Conventional open lumbar discectomy performed as repeat surgery for recurrent herniation showed satisfactory results. Based on the results of this study, repeat discectomy can be recommended for the management of recurrent lumbar disc herniation. Further study is needed to evaluate factors related to the outcomes of repeat discectomy.

Key Words: Discectomy $\cdot$ Lumbar spine $\cdot$ Recurrent

\section{INTRODUCTION}

Recurrent disc herniation has been reported in $5-15 \%$ of patients after disc excision, which represents the most common cause of unsatisfactory results ${ }^{1-3,5,7,9-12,15)}$. The recurrence of symptoms after an initial period of symptomatic relief can be caused by a recurrent disc herniation, new disc herniation at a different disc level, epidural fibrosis, arachnoiditis, foraminal stenosis, or segmental instability ${ }^{9,14)}$.

Several conditions have been termed as "recurrent disc herniation" including recurrence at the same disc level and on the ipsilateral side as the primary herniation, contralateral herniation at the same disc, or a new herniation at a different

- Received: January 16, 2012 - Revised: March 15, 2012

- Accepted: March 26, 2012

Corresponding Author: Young Min Kwon, MD

Department of Neurosurgery, Dong-A University College of Medicine,

Dongdaesin-dong 3-ga, Seo-gu Busan 602-715, Korea

Tel: +82-51-240-5241, Fax: +82-51-242-6714

E-mail: ymkwon@dau.ac.kr level ${ }^{3)}$. The clinically appropriate definition of "recurrent disc herniation" is that a disc herniation at the same disc level with an ipsilateral side as a previously operated on. There have been numerous studies of recurrent disc herniation, but these have analyzed mixed patient populations and included patients with different causes of failed back surgery, as well as those with herniation at a different level or contralateral side compared with the primary discectomy.

We designed this study to analyze the factors influencing the clinical outcome and efficiency of repeat discectomy for recurrent lumbar disc herniation at the same level and on the ipsilateral side after primary discectomy.

\section{MATERIAL AND METHODS}

From January 1990 to September 2011, a series of 2816 patients underwent lumbar discectomy for disc herniation. Of these patients, 52 patients underwent on repeat discectomy due to recurrent disc herniation. Clinical and radiologic data of these 52 patients were retrospectively reviewed. All opera- 
tions were performed according to the standard pattern proposed by the senior author. Recurrent lumbar disc herniation was defined as disc herniation at the same level and on the ipsilateral side. The symptomatic relief period after the primary discectomy should be more 4 weeks. The patients with a disc herniation occurring at a new level or contralateral side with respect to the primary discectomy were excluded from this study.

Clinical and radiological factors associated with recurrent disc herniation were evaluated using preoperative clinical medical charts and radiological examinations. The evaluated demographic and clinical parameters were as follows: age, sex, smoking habit, diabetes mellitus (DM), traumatic event, symptom duration before repeat discectomy, and time to repeat discectomy from initial discectomy.

The extent of disc herniation was evaluated and classified as protrusion, extrusion or sequestration. A disc was considered protruded if the greatest plane, in any direction between the edges of the disc material beyond the disc, was less than the distance between the edges of the base when measured in the same plane. A disc was considered extruded if, in at least in one plane, any one distance between the edges of the disc material beyond the disc space was greater than the distance between edges of the base measured in the same plane. A sequestrated disc was one in which the disc material was clearly separate from the originating disc $^{2)}$. Magnetic resonance imaging assessments were performed independently by a neuroradiologist and a neurosurgeon.

The pain was evaluated by a 10-point visual analogue scale (VAS) scoring (0-10) before and after repeat discectomy, and differences of VAS score were measured (VAS). Postoperative satisfaction was evaluated using Macnab criteria (Excellent, Good, Fair and Poor). Clinical success was defined as if patients showed (1) more than 4 point improvement in VAS scores, (2) excellent or good in Macnab criteria and (3) no major complication related to the surgery. Pre- and postoperative data were assessed using clinical charts and surgical reports.

Statistical analysis was done using Fisher's exact test or the Cochran-Mantel-Haenszel test for categorical variables, anda $\mathrm{t}$-test for continuous variables. All $\mathrm{p}$-values less than 0.05 were considered statistically significant. All statistical analyses were performed using SAS 9.1.3 statistical software.

\section{RESULTS}

The group consisted of 35 men and 17 women and their average age at repeat discectomy was 40.4 years (range 18-72 years) (Table 1). The level of recurrent disc herniation was
L4/5 in 31 cases, L5/S1 in 17 cases, L3/4 in two cases. The symptoms and sign are summarized in Table 2. All patients had back pain and sciatica after a pain free interval following discectomy.

Eight patients experienced recurrent symptoms after a traumatic event. The mechanism of onset was a car accident in 4 , a sporting accident in 2 , and a fall in 2 . The remaining 45 patients had no definite history of injury. The incidence of chronic smoking was 8 (15.4\%), and of diabetes mellitus $2(3.8 \%)$. The time from prior surgery to the repeat discectomy averaged 24 months (Table 1 ). The average symptom duration the before repeat discectomy is 2.5 months.

The outcome of postoperative clinical symptoms was evaluated using Macnab criteria, and the results were as follows: Excellent (28), Good (22), Fair (1), and Poor ( $\mathrm{n}=1)$. The patient with grade poor had a post-operative infection, and required revisional surgery. The patient with grade fair required further pain relief such as pain block. The mean preoperative VAS score in revision surgery was 6.98, and this improved after the revision surgery to 2.1. Forty-seven patients out of 52 showed improvement of more than 4-point on VAS scale. Thus, clinical success was seen in 47 of 52 patients (90\%).

No factors affected clinical outcome of repeat discectomy for recurrent disc herniation assessed by Macnab criteria and VAS score according by age, sex, diabetes mellitus, smoking,

Table 1. Demographics of patient

\begin{tabular}{ll}
\hline \hline Variable & \\
\hline Number of patients & 52 \\
Age (years) & 40.4 (18-72) \\
Sex (male:female) & $35: 17$ \\
Smoking status (\%) & 15.4 \\
Diabetes mellitus (\%) & 3.8 \\
Operation time interval (months) & $24.3(3-240)$ \\
Symptom duration (months) & $2.6(0.2-18)$ \\
The extent of disc herniation (\%) & \\
$\quad$ protrusion & 57.7 \\
$\quad$ extrusion & 36.5 \\
$\quad$ sequestration & 5.8 \\
\hline
\end{tabular}

Table 2. Symptoms and signs in patients with recurrent disc herniation $(n=52)$

\begin{tabular}{lc}
\hline \hline Symptoms and signs & $\mathrm{n}$ \\
\hline Back pain & 52 \\
Sciatica & 52 \\
Lumbar tenderness & 43 \\
Positive straight-leg raising test & 8 \\
Motor weakness & 4 \\
Sensory deficit & 6 \\
Bladder dysfunction & 0 \\
\hline
\end{tabular}


Table 3. Influencing factors and clinical outcomes (Macnab criteria)

\begin{tabular}{|c|c|c|c|c|c|c|}
\hline & & \multicolumn{4}{|c|}{ Macnab criteria } & \multirow[b]{2}{*}{$p$-value } \\
\hline & & Excellent $(n=28)$ & Good $(n=22)$ & Fair $(n=1)$ & Poor $(n=1)$ & \\
\hline Age (years) & & $40(18-62)$ & $41(21-72)$ & 72 & 21 & 0.873 \\
\hline Sex & $\begin{array}{l}\text { Female } \\
\text { male }\end{array}$ & $\begin{array}{r}8(28.6) \\
20(71.4)\end{array}$ & $\begin{array}{r}8(36.4) \\
14(63.6)\end{array}$ & $\begin{array}{l}1(100) \\
0(0.0)\end{array}$ & $\begin{array}{l}0(0.0) \\
1(100)\end{array}$ & 0.589 \\
\hline Smoking status & $\begin{array}{l}\text { yes } \\
\text { no }\end{array}$ & $\begin{array}{r}6(21.4) \\
22(78.6)\end{array}$ & $\begin{array}{c}2(9.1) \\
20(90.9)\end{array}$ & $\begin{array}{l}0(0.0) \\
1(100)\end{array}$ & $\begin{array}{l}0(0.0) \\
1(100)\end{array}$ & 0.197 \\
\hline Operation interval (months) & & $24(1-168)$ & $23(1-240)$ & 91 & 2 & 0.716 \\
\hline Symptom duration (months) & & $3(0-18)$ & $3(0-11)$ & 2 & 1 & 0.956 \\
\hline Traumatic event & $\begin{array}{l}\text { yes } \\
\text { no }\end{array}$ & $\begin{array}{r}4(14.3) \\
24(85.7)\end{array}$ & $\begin{array}{r}4(18.2) \\
18(81.8)\end{array}$ & $\begin{array}{l}0(0.0) \\
1(100)\end{array}$ & $\begin{array}{l}0(0.0) \\
1(100)\end{array}$ & 0.926 \\
\hline
\end{tabular}

"p-values were derived from + test for testing the mean difference between "Excellent" and "Good" groups due to the small sample size in "Fair" and "Poor" groups ( $\mathrm{n}=1$, respectively).

Table 5. Clinical outcomes and the extent of disc herniation

\begin{tabular}{|c|c|c|c|c|c|}
\hline & \multicolumn{3}{|c|}{ The extent of disc herniation } & \multirow[b]{2}{*}{$\mathrm{p}$-value } \\
\hline & & $\begin{array}{l}\text { Protrusion } \\
(n=30)\end{array}$ & $\begin{array}{c}\text { Extrusion } \\
(n=19)\end{array}$ & $\begin{array}{l}\text { Sequestration } \\
\qquad(n=3)\end{array}$ & \\
\hline \multirow{4}{*}{$\begin{array}{l}\text { Macnab } \\
\text { criteria }\end{array}$} & Excellent & 19 (63.3) & $8(42.1)$ & $1(33.3)$ & \multirow{4}{*}{0.469} \\
\hline & Good & $9(30.0)$ & $11(57.9)$ & $2(66.7)$ & \\
\hline & Fair & $1(3.3)$ & $0(0.0)$ & $0(0.0)$ & \\
\hline & Poor & $1(3.3)$ & $0(0.0)$ & $0(0.0)$ & \\
\hline$\triangle \mathrm{VAS}$ & $\square 4$ & $27(90.0)$ & $18(95.0)$ & $2(66.7)$ & \multirow{2}{*}{0.284} \\
\hline score & $<4$ & $3(10.0)$ & $1(5.0)$ & 1 (33.3) & \\
\hline
\end{tabular}

Table 4. Influencing factors and clinical outcome (VAS score)

\begin{tabular}{lcccc}
\hline \hline & & \multicolumn{2}{c}{ VAS score } & \\
\cline { 3 - 4 } & & $\geqq 4(\mathrm{n}=47)$ & $<4(\mathrm{n}=5)$ & p-value \\
\hline Age (years) & $41(21-72)$ & $37(18-58)$ & 0.592 \\
Sex & Female & $16(34)$ & $1(20)$ & 1.000 \\
& male & $31(66)$ & $4(80)$ & \\
Smoking status & yes & $7(15)$ & $1(20)$ & 1.000 \\
& no & $40(85)$ & $4(80)$ & \\
Operation interval & $25(1-240)$ & $18(1-77)$ & 0.721 \\
(months) & & & & \\
Symptom duration & & & & \\
(months) & & & & \\
Traumatic event & yes & $5(11)$ & $3(60)$ & 0.212 \\
& no & $42(89)$ & $2(40)$ & 0.022 \\
\hline
\end{tabular}

time interval between repeat and primary discectomy, symptom durationbefore the repeat discectomy, and extent of disc herniation. Traumatic event showed significantly affected the VAS score, however not significantly on the Macnab criteria. According to clinical success, traumatic event was the only
Table 6. Clinical outcomes and operation time interval

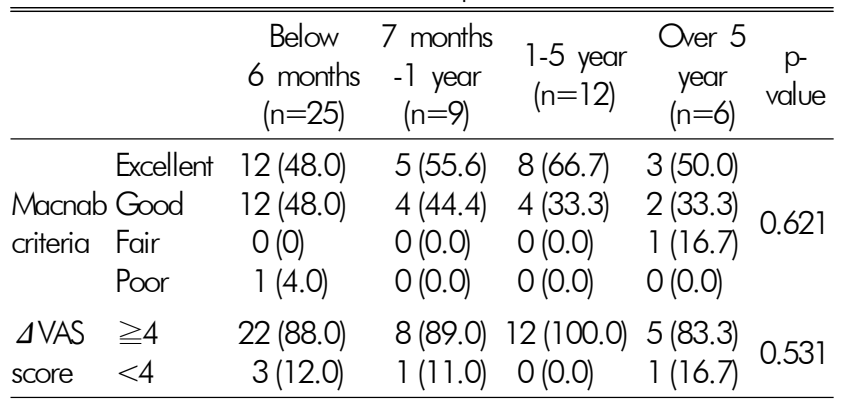

statistically significant factor that showed poor clinical outcome after repeat discectomy $(\mathrm{p}=0.022)$ (Table $3,4,5,6)$.

\section{DISCUSSION}

Conventional open lumbar discectomy has been used as a surgical treatment for a herniated lumbar disc since the initial ofthis discovery disease entity more than 70 years ago ${ }^{10)}$. Primary lumbar discectomy usually results a good clinical outcome. However, the revision rate has ranged between 2-19\% in previous studies ${ }^{49}$ ). There are numerous operative methods that can be done on patients with recurrent lumbar disc herniation such as repeat discectomy, percutaneous endoscopic discectomy, posterior lumbar interbody fusion and transforaminal interbody fusion. The current study assessed patients with proven recurrent disc herniation at the same location as a previous surgically treated herniation. Patients with disc herniation at the new segment, contralateral side herniation were excluded. Therefore, the authors were able to study the clinical outcomes after revision surgery for a true recurrent disc herniation. 
It has been shown that the outcomes of patients surgically treated for recurrent disc herniation vary widely. This may be related to the different inclusion criteria applied in these studies. Patients with recurrent herniation are often reviewed together with those presenting with herniation occurring at a new level or with those in whom lumbar surgery has failed for another reason. Cinotti et al. ${ }^{3)}$ prospectively analyzed patients with recurrent disc herniation at the same level as at primary discectomy, either on the same or opposite site, and found no significant difference in clinical outcome between patients with recurrentherniation and those without recurrent herniation. In another study, Suk et al. ${ }^{14)}$ showed that satisfactory results were obtained when conventional open discectomy was used for revision surgery for recurrent lumbar disc herniation, and that these were comparable to those obtained with primary discectomy. Erbayraktar et al. ${ }^{4)}$ reported the findings of repeat discectomy in 22 patients with prior lumbar discectomy at the same or a different level, either on the same or on the opposite site. They found an overall clinical improvement is $91 \%$ of patients who underwent repeat surgery. Satisfactory outcomes were also obtained in the currentstudy. There were 28 excellent, 22 good, 1 fair and 1 poor Macnab criteria. Clinical success was seen in 47 of the 52 patients (90\%).

In patients with diabetes mellitus, hospitalization is prolonged and there is a higher risk of postoperative infection as well as poorer results ${ }^{15)}$. Simpson et al. ${ }^{13)}$ reported that excellent and good outcomes in 39\% of their patients with diabetes, while $95 \%$ of those without diabetes, after an initial discectomy. The patients with diabetes has poor outcome caused by diabeticpolyneuropathy before neural compression caused by disc disease. However, in our study, diabetes was not a statistically significant factor affecting clinical outcome.

Predisposing factors associated with an increased risk of primary disc herniation are a constitutional weakness of the annular tissue, exposure to repetitive lifting, vibrations and smoking. Isolated trauma or injury seems to play a prominent role inthe etiology of disc herniation since this has been reported by $0.2-10.7 \%$ of adults with documented herniation ${ }^{3)}$. We found that $15 \%$ of patients with recurrent herniation related the onset of radicular pain to traumatic event. This suggests that the annular incision performed at surgery makes the disc to be more susceptible to sudden prolapse, particularly under conditions of mechanical overload such as those occur during sports or lifting. In the present study, traumatic events were statistically significant factor affecting VAS score, but not a statistically significant factor affecting Macnab criteria. According to clinical success criteria in this study, traumatic events are the statistically significant factor for poor clinical outcome $(p<0.05)$. That was VAS score puts emphasis on subjective pain, combined musculoskeletal pain due to trauma. In Macnab criteria, good was thatpatients have nonneuropathic pain, but postoperative pain was relieved. Thus, although pain due to disc disease was relieved, VAS score not decreased.

Morgan-Hough et al. ${ }^{12)}$ noted that patients with protrusion are almost three times as likely to require revision surgery asthose with extrusions or sequestrations. This finding could be because a protrusion represents the beginning of a process of serial fragmentation of disc material, whereas extrusion and sequestration occur at the end stages of this process. However, in our study, the extent of disc herniation was not a statistically significant factor affecting clinical outcome.

There are many reports that analyzed the outcomes of repeat surgery in relation to the time interval between the primary and second operation. Some reported that shorter period of time (less than one year) between primary and second operation results the better clinical outcomes ${ }^{4,8)}$. However, Fandifio et al. ${ }^{5)}$ reported that the clinical outcomes were independent of the time interval between the primary and repeat surgery, which showed the same result in this current study.

Different factors such as patient's age, sex, smoking, traumatic events, operation time interval, symptom duration before the repeat discectomy that may influence the outcome of repeat discectomy for recurrent disc herniation have beenanalyzed in numerous studies, but still remain controversial. Some reports found no significant factors that influence the clinical outcomes after the repeat lumbar discectomy ${ }^{11,14)}$.

Some aspects of the current study design might have affected the validity and thus the results. The patients enrolled in this series were transferred from different hospitals. They might have undergone primary surgeries for different indication, and different procedures might have been used. In addition, no factors were found to be statistically related to the outcome of repeated discectomy for recurrent disc herniation ( $p>0.05$ ) except for traumatic events. The strength of this finding might be weak; that is the insignificant results of the other factors might have been due to an insufficient numbers of patients for detecting significant differences. More research was needed on the dural tear caused by epidural adhesion that important role in evaluating outcome of revision surgery. Furthermore follow up period was relatively short. Therefore, results of the patients may not have been fully evaluated.

\section{CONCLUSION}

Conventional open lumbar discectomy performed as a revision surgery for recurrent herniation showed satisfactory results. Based on the results of this study, repeat discectomy can be recommended for the management of recurrent lumbar 
disc herniation.However, patients with traumatic event before revision seem to show poor clinical outcome. Further study is required to identify factors associated with the outcome of revision surgery for recurrent disc herniation.

\section{REFERENCES}

1. Baba H, hen Q, Kamitani K, Imura S, Tomita K: Revision surgery for lumbar disc herniation An analysis of 45 patients. International Orthopaedics 19:98-102, 1995

2. Choi KB, Lee DY, Lee SH: Contralateral Reherniation after Open Lumbar Microdiscectomy: A Comparison with Ipsilateral Reherniation. J Korean Neurosurg Soc 44:320-326, 2008

3. Cinotti G, Roysam GS, Eisenstein SM, Postacchini F: Ipsilateral recurrent lumbar disc herniation. A prospective, controlled study. J Bone Joint Surg Br 80:825-832, 1998

4. Erbayraktar S, Acar F, Tekinsoy B, Acar Ü, Metin GE: Outcome Analysis of Reoperations after Lumbar Discec- tomies: a Report of 22 Patients. J Med Sci 48:33-41, 2002

5. Fandifio J, Botana C, VUadrich A, Gomez-Bueno J: Reoperation After Lumbar Disc Surgery: Results in 130 Cases. Acta Neurochir 122:102-104, 1993

6. Gaston P, Marshall RW: Survival analysis is a better estimate of recurrent disc herniation. J Bone Joint Surg Br 85:535-537, 2003

7. Häkkinen A, Kiviranta I, Neva Marko H, Kautiainen H, Ylinen
J: Reoperations after first lumbar disc herniation surgery; a special interest on residives during a 5-year follow-up. BMC Musculo skeletal Disorders 8:2, 2007

8. Kayaoglu CR, Calikoglu C, Binler S: Re-operation after Lumbar Disc Surgery: Results in 85 Cases. The Journal of International Medical Research 31:318-323, 2003

9. Kim HH, Kwon YJ, Won YS, Yang JY, Choi ChS: Clinical Analysis of Prognostic Factors of Reoperation after Degenerative Lumbar Disc Surgery. Korean J Spine 2(2):133-137, 2005

10. Koebbe Christopher J, Maroon Joseph C, Abla Adnan, El-Kadi Hikmat: Lumbar microdiscectomy: a historical perspective and current technical considerations. Neurosurg Focus 13(2):3, 2002

11. Li-Yang D, Qing Z, Wei-Fang Y, Lei S: Recurrent lumbar disc herniation after discectomy: outcome of repeat discectomy. Surgical Neurology 64:226-231, 2005

12. Morgan-Hough CV, Jones PW, Eisenstein SM: Primary and revision lumbar discectomy. A 16-year review from one centre. J Bone Joint Surg Br 85:871-874, 2003

13. Simpson JM, Silveri CP, Balderston RA, Simeone FA, An HS: The results of operations on the lumbar spine in patients whohave diabetes mellitus. J Bone Joint Surg Am 75:1823-1829, 1993

14. Suk KS, Lee HM, Moon SH, Kim NH: Recurrent lumbar disc herniation: Results of operative management. Spine 26:672676, 2001

15. Swartz KR, Trost GR: Recurrent lumbar disc herniation. Neurosurg Focus 15:10, 2003 